\title{
Capabilities, limitations, and misconceptions of using OCT to assess vulnerable plaques
}

Mark E. Brezinski

Serious concerns exist about the Review by Otsuka et al. (Clinical classification of plaque morphology in coronary disease. Nat. Rev. Cardiol. 11, 379-389; 2014), ${ }^{1}$ some of which are discussed in this Correspondence. The concerns are predominately about the authors' understanding of the principles and application of optical coherence tomography (OCT), including factors that affect imaging penetration and acquisition rate, the difference between lipid collections and necrotic cores, and the use of OCT to detect macrophages.

On page 1, the authors state: "Of note, the very high resolution of OCT restricts the depth of penetration of the light beam through blood and tissue (to $1-3 \mathrm{~mm}$ ), resulting in the incomplete reflection from the deeper arterial layers." ${ }^{\text {The }}$ reduced penetration is not caused by the resolution, but predominately by the amount of photon scattering; a detailed analysis of the factors affecting penetration has been published previously. ${ }^{2}$ Two examples illustrate this point. In the eye-a low scattering tissuelight-beam penetration is on the order of centimetres at the same resolution as in blood vessels. Moreover, we have demonstrated that OCT cannot usually penetrate blood because of the high scattering caused by the difference in refractive index between serum and the cytoplasm of red blood cells (refractive index mismatch also causes the high scattering between lipidbased and water-based tissue). ${ }^{3}$ By increasing the refractive index of serum, we have made blood almost transparent, thereby eliminating scattering. ${ }^{3}$ This point is critical to interpreting plaque images, because the pronounced mismatch produced by small lipid collections and calcium clusters (but not macrophages) leads to high scattering, diffuse borders, and rapid attenuation. Techniques exist, such as the use of a parallel continuous wave ultrasound beam or elastography, to overcome these effects.
A glaring inconsistency throughout the Review is that the authors sometimes treat lipid collections and necrotic cores as nearly identical, and at other times as distinct entities. The OCT literature has been focused primarily on the identification of lipid in plaques, whereas histopathology studies have been focused on identifying necrotic cores. No study has been conducted to compare the two, and yet the authors use the terms interchangeably. This point is important for interpreting plaque composition.

The authors' description of the use of OCT to detect macrophages is inconsistent and, at points, unsubstantiated claims are made. The concept that OCT can be used to measure macrophages and foam cells with a simplistic image-processing technique (and not by directly visualizing them) is based on one paper, ${ }^{4}$ whereas we have repeatedly questioned the validity of these data. ${ }^{2,5,6}$ Another group has verified our claims. ${ }^{7}$ However, the authors seem not to appreciate this point, as demonstrated from Figures 2-5, in which they attribute large dropout areas in the image to macrophage localization. Plaque macrophages are not sufficiently numerous, nor does sufficient refractive index mismatch exist, to account for these large attenuations. The dropout areas are likely to be caused by lipid collections, which are abundant in the corresponding histopathology and have large refractive index mismatches (see Figure 1 in our previous publication $\left.^{6}\right)$. Further emphasizing their confusion on the topic, on page 2 the authors state: "Detection of macrophage accumulation in atherosclerotic plaques might also be possible." However, later on the same page, they state: "When macrophages are present in the more-superficial intimal layers, signal-rich, distinct, or confluent punctate areas with shadowing of the underlying tissue structures are observed via OCT." "This assertion is also made in the legends of Figures 2-5. No published work supports the idea that the presence of macrophages leads to shadowing with OCT.

On page 1 , the authors state that the spectral domain technique "allows the backscattering light from various locations to be measured simultaneously, enabling a rapid pullback and shorter acquisition time". ${ }^{1}$ These techniques actually measure the frequency spectrum of an A-scan and create the backscattering profile through signal processing (by Fourier transform). They do not measure backscattering simultaneously at various depths. The high acquisition rates are produced because spectral techniques contain no moving parts (unlike the time domain system), and instead use computer processing, but not by sampling at different depths simultaneously.

Finally, the statement is made, but not referenced, that "plaque rupture is the underlying substrate in at least two-thirds of individuals who have acute coronary syndrome, and usually occurs in plaques with a large necrotic core and a thin, but substantially inflamed, fibrous cap." ${ }^{1}$ I am not aware of any data to show that plaques predisposed to rupture are substantially inflamed. Systemic inflammation is well documented as a cause of atherogenesis and, after rupture, the plaque is both inflamed and highly thrombogenic. ${ }^{8}$ However, the concept that plaques are inflamed just before rupture is speculation.

These concerns, I feel are the most relevant to the article. For these reasons, I feel that the Review by Otsuka, et al. might mislead readers as to the current state of cardiovascular OCT and how the field might develop.

Brigham and Women's Hospital, 75 Francis Street, MRB-114, Boston, MA 02467, USA. mebrezin@mit.edu

Competing interests

The author declares no competing interests. 


\section{CORRESPONDENCE}

1. Otsuka, F., Joner, M., Prati, F., Virmani, R. \& Narula, J. Clinical classification of plaque morphology in coronary disease. Nat. Rev. Cardiol. 11, 379-389 (2014).

2. Brezinski, M. E. Optical Coherence Tomography: Principles and Applications (Academic Press, 2006).

3. Brezinski, M., Saunders, K., Jesser, C., Li, X. D. \& Fujimoto, J. Index matching to improve optical coherence tomography imaging through blood. Circulation 103, 1999-2003 (2001).
4. Tearney, G. J. et al. Quantification of macrophage content in atherosclerotic plaques by optical coherence tomography. Circulation 107, 113-119 (2003).

5. Brezinski, M. E. Applications of optical coherence tomography to cardiac and musculoskeletal diseases: bench to bedside? J. Biomed. Opt. 12, 051705 (2007).

6. Brezinski, M. E. Current capabilities and challenges for optical coherence tomography as a high-impact cardiovascular imaging modality. Circulation 123, 2913-2915 (2011).

7. Phipps, J. E., Vela, D., Hoyt, T., Milner, T. \& Feldman, M. Macrophages are not the only source of bright spots in intravascular optical coherence tomography images: a quantitative analysis of human atherosclerosis [abstract]. J. Am. Coll. Cardiol. 63 (Suppl.), A1754 (2014).

8. Buffon, A. et al. Widespread coronary inflammation in unstable angina. N. Engl. J. Med. 347, 5-12 (2002) 